\title{
Pattern of lung function decline in patients with amyotrophic lateral sclerosis: implications for timing of noninvasive ventilation
}

\author{
Tanmay S. Panchabhai ${ }^{1}$, Eduardo Mireles Cabodevila (10² , Erik P. Pioro (1) ${ }^{3}$, \\ Xiaofeng Wang ${ }^{4}$, Xiaozhen $\mathrm{Han}^{4}$ and Loutfi S. Aboussouan (103)
}

Affiliations: ${ }^{1}$ Section of Interventional Pulmonology, Beth Israel Deaconess Medical Center, Boston, MA, USA ${ }^{2}$ Dept of Pulmonary Medicine, Respiratory Institute, Cleveland Clinic, Cleveland, OH, USA. ${ }^{3}$ Dept of Neurology, Neurological Institute, Cleveland Clinic, Cleveland, $\mathrm{OH}$, USA. ${ }^{4}$ Dept of Qualitative Health Sciences, Lerner Research Institute, Cleveland Clinic, Cleveland, $\mathrm{OH}$, USA.

Correspondence: Loutfi S. Aboussouan, Dept of Pulmonary Medicine, Respiratory Institute, Cleveland Clinic, 9500 Euclid Avenue, A 90, Cleveland, OH 44195, USA. E-mail: aboussläccf.org

\section{ABSTRACT}

Background: The course of lung function decline in amyotrophic lateral sclerosis (ALS) and the effect of noninvasive positive-pressure ventilation (NIPPV) on that decline are uncertain. We sought to model lung function decline, determine when NIPPV is initiated along that course, and assess its impact on the course of decline.

Methods: An observed sigmoid pattern of forced vital capacity decline was reproduced with a four-parameter nonlinear mixed-effects logistic model.

Results: Analyses were performed on 507 patients overall and in 353 patients for whom a determination of adherence to NIPPV was ascertained. A sigmoid bi-asymptotic model provided a statistical fit of the data and showed a period of stable vital capacity, followed by an accelerated decline, an inflection point, then a slowing in decline to a plateau. By the time NIPPV was initiated in accordance with reimbursement guidelines, vital capacity had declined by $\geqslant 85 \%$ of the total range. Nearly half of the total loss of vital capacity occurred over 6.2 months centred at an inflection point occurring 17 months after disease onset and 5.2 months before initiation of NIPPV at a vital capacity of about $60 \%$. Fewer bulbar symptoms and a faster rate of decline of lung function predicted adherence to NIPPV, but the intervention had no impact on final vital capacity.

Conclusions: In patients with ALS, vital capacity decline is rapid but slows after an inflection point regardless of NIPPV. Initiating NIPPV along reimbursement guidelines occurs after $\geqslant 85 \%$ of vital capacity loss has already occurred.

@ERSpublications

In patients with amyotrophic lateral sclerosis, vital capacity decline is rapid but slows after an inflection point regardless of NIPPV. Initiation of NIPPV according to guidelines occurs when $>\mathbf{8 5} \%$ of vital capacity loss has already occurred. http://bit.ly/2JOiEZG

Cite this article as: Panchabhai TS, Mireles Cabodevila E, Pioro EP, et al. Pattern of lung function decline in patients with amyotrophic lateral sclerosis: implications for timing of noninvasive ventilation. ERJ Open Res 2019; 5: 00044-2019 [https://doi.org/10.1183/23120541.00044-2019].

This article has supplementary material available from openres.ersjournals.com

Received: 25 March 2019 | Accepted after revision: 20 July 2019

Copyright $\odot E R S$ 2019. This article is open access and distributed under the terms of the Creative Commons Attribution Non-Commercial Licence 4.0. 


\section{Introduction}

Amyotrophic lateral sclerosis (ALS) is a neuromuscular disorder characterised by respiratory muscle weakness and a progressive decline in lung function [1]. Survival is reduced by bulbar dysfunction, frontotemporal dementia, older age, rapid progression, C9orf72 gene repeat expansion, diagnostic delay and low forced vital capacity [2]. Death occurs within 3 to 5 years of onset, generally from respiratory complications [3, 4]. Disease-modifying pharmacologic options have not paralleled advances in the understanding of the pathogenesis and molecular genetics of ALS and consist of riluzole and edaravone with limited survival benefits [3]. In contrast, noninvasive positive-pressure ventilation (NIPPV) prevents or delays tracheostomy $[1,5]$ and significantly improves survival and quality of life $[6,7]$.

Indications and recommendations for the timing of NIPPV vary and are mostly driven by reimbursement guidelines in the USA $[1,5,8,9]$. ALS practice recommendations allow criteria such as symptoms and sniff nasal pressure $[1,5,9,10]$, with a higher vital capacity cut-off in some European guidelines $[1,9]$. Uncertainty remains about the course of lung function decline, which is variably characterised as accelerated [11], steady [12-14] or decelerating [15-17]. Others attribute the deceleration of decline to NIPPV [5, 18-21]. Resolving these issues is of utmost importance to inform the timing of NIPPV.

We therefore studied a cohort of patients with ALS, aiming to: 1) model the course of lung function decline; 2) assess the point in decline at which NIPPV is initiated, based on current guidelines; and 3) compare the pattern of decline in individuals who adhere to NIPPV with those who do not.

\section{Methods}

\section{Study design and data collection}

The Institutional Review Board of the Cleveland Clinic approved this study in accordance with the amended Declaration of Helsinki (approval 10-428). Informed consent was not required for this type of study. ALS was diagnosed by neurologists in the Section of ALS and Related Disorders Clinic using World Federation of Neurology El Escorial criteria [22]. Trained neurology clinic personnel obtained spirometry and a functional assessment (revised ALS Functional Rating Scale (ALSFRS-R)) [23] every 3 to 6 months. Patients were then referred to the pulmonary neuromuscular clinic at the onset of dyspnoea on exertion, orthopnoea or a per cent predicted forced vital capacity $(\mathrm{FVCP})<60 \%$. The pulmonary neuromuscular clinic then performed a clinical assessment, obtained an FVCP and per cent predicted maximum inspiratory pressure (MIPP) and re-evaluated those parameters every 3 to 6 months. Sniff inspiratory nasal pressure (SNIP) and arterial blood gases were also obtained (see supplement). All patients with probable or definitive ALS (defined by World Federation of Neurology El Escorial criteria [22]) referred to the neuromuscular pulmonary clinic between September 1, 2003 and January 31, 2014 were included in this study.

\section{Pulmonary function tests}

Spirometry was performed with a Puritan-Bennett PB-100 spirometer (Puritan-Bennett, Overland Park, KS, USA) in the Section of ALS and Related Disorders Clinic, and in a certified pulmonary function laboratory in the pulmonary clinic. Leak-control methods in patients with bulbar disease included a large mouthpiece with a flange positioned between the teeth and lips, mouthpieces with a tongue-positioning ridge (FreeFlow $^{\mathrm{TM}}$, Vyaire Medical), nose clips and manual lip sealing. Standardised spirometry techniques with predicted values from the Third National Health and Nutrition Examination Survey were applied [24, 25]. Within the limitations of fatigue imposed by the disease, we aimed to obtain at least three forced vital capacity manoeuvres that met defined acceptability criteria [25]. Maximum inspiratory pressures were measured from the residual volume using an inspiratory force meter (Boehringer, Ingelheim am Rhein, Germany) and with the largest pressure maintained for at least $1 \mathrm{~s}$ reported using predicted values from Black and Hyatt $[26,27]$. For those unable to maintain a plateau of at least $1 \mathrm{~s}$, peak pressure was reported.

\section{Noninvasive ventilation and respiratory assistance devices}

NIPPV was initiated for FVCP $\leqslant 50 \%$, or hypercapnia $\left(P_{\mathrm{AO} 2} \geqslant 45 \mathrm{mmHg}\right)$, consistent with Local Coverage Determinations from the Centers for Medicare and Medicaid Services [8]. For patients with orthopnoea, dyspnoea, nonrestorative sleep, morning headaches or daytime sleepiness, NIPPV was initiated for a MIPP $\leqslant 60 \mathrm{cmH}_{2} \mathrm{O}$ or if nocturnal pulse oximetry demonstrated an oxygen saturation $\leqslant 88 \%$ for $\geqslant 5$ min of recording time [8]. NIPPV consisted of pressure-limited devices in spontaneous-timed mode with an initial inspiratory pressure of $10-15 \mathrm{cmH}_{2} \mathrm{O}$ (to patient comfort) and a low expiratory pressure, generally $5 \mathrm{cmH}_{2} \mathrm{O}$ (or the known pressure that controlled apnoea in previously diagnosed sleep apnoea). Patients were instructed to use NIPPV during the entire sleep period and as necessary in the daytime. Comfort was optimised by interface selection, adjustment of device settings, nasal steroid sprays for nasal congestion and cough-assist and suction devices for secretion clearance. Adherence, defined as the ability to sleep with the device for at least 4 hours consecutively during a 24-hour period, was ascertained at follow-up 
visits and documented in medical records. Final determination of adherence was made by consensus of three physicians (TSP, EMC, LSA) after record review.

\section{Mathematical modelling and statistical analysis}

Two plots informed parametric model selection: FVCP decline from disease onset for patients with a higher number of observations (figure 1), and a nonparametric regression curve of FVCP decline data for the subset of patients in whom NIPPV was initiated, with the course centred at the initiation time (figure 2) [28]. Both plots indicated a sigmoid pattern of FVCP change with an initial plateau, accelerated decline, inflection point, decelerating decline and terminal plateau.

A four-parameter nonlinear mixed-effects logistic model was used to reproduce this bi-asymptotic course and solve for the individual parameters (figure 3) [29]

$$
\mathrm{FVCP}=b_{1}+\frac{b_{2}}{1+\mathrm{e}^{\frac{b_{3}-t}{b_{4}}}}
$$

where $t$ is time referenced either from the first onset of disease for all patients (Model 1), or from initiation of NIPPV and stratified by NIPPV adherence (Model 2). In both models, $b_{1}$ is the FVCP before the onset of lung function decline, and $b_{2}$ is the magnitude of decline to the lower asymptote, introduced as a negative number so that $b_{1}+b_{2}$ represents the FVCP at the lower asymptote. The time parameters, $b_{3}$ and $b_{4}$, are expressed in months, with $b_{3}$ as the time from the reference to the midpoint between the two asymptotes, also signalling the inflection point that separates an accelerating from a decelerating FVCP decline. The time scale factor, $b_{4}$, reflects the speed of FVCP decline, with smaller values representing a faster decline.
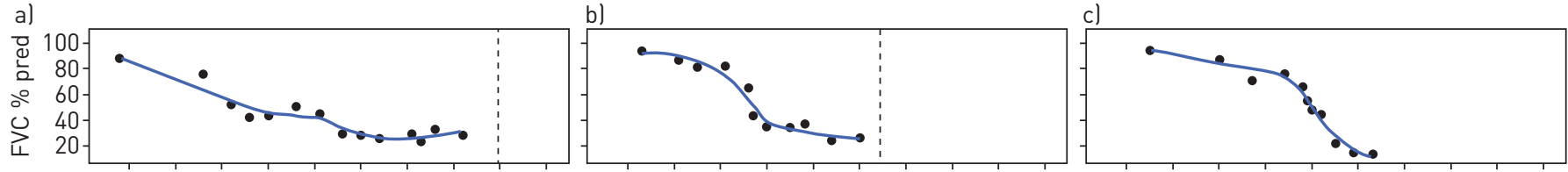

d) e)
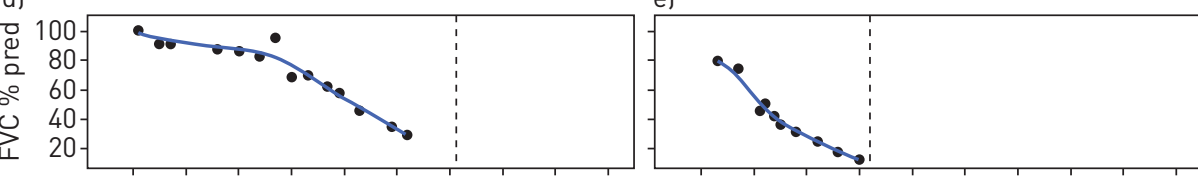

f)
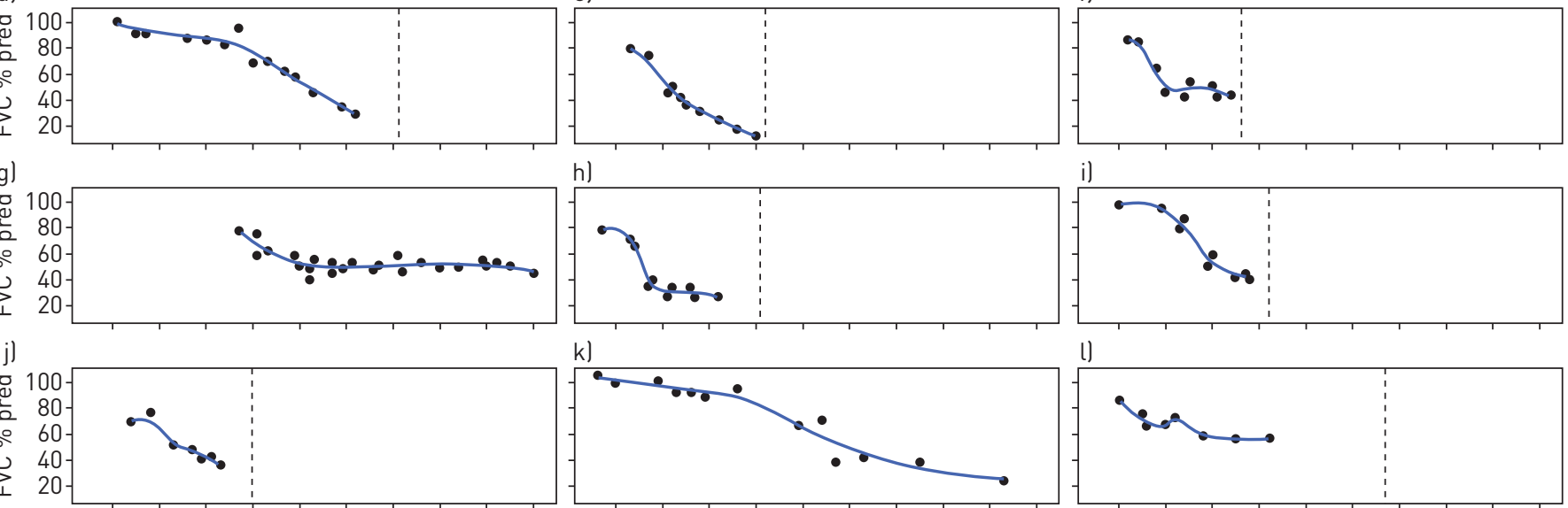

m)

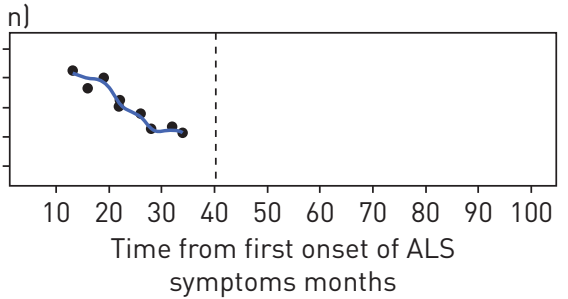

()
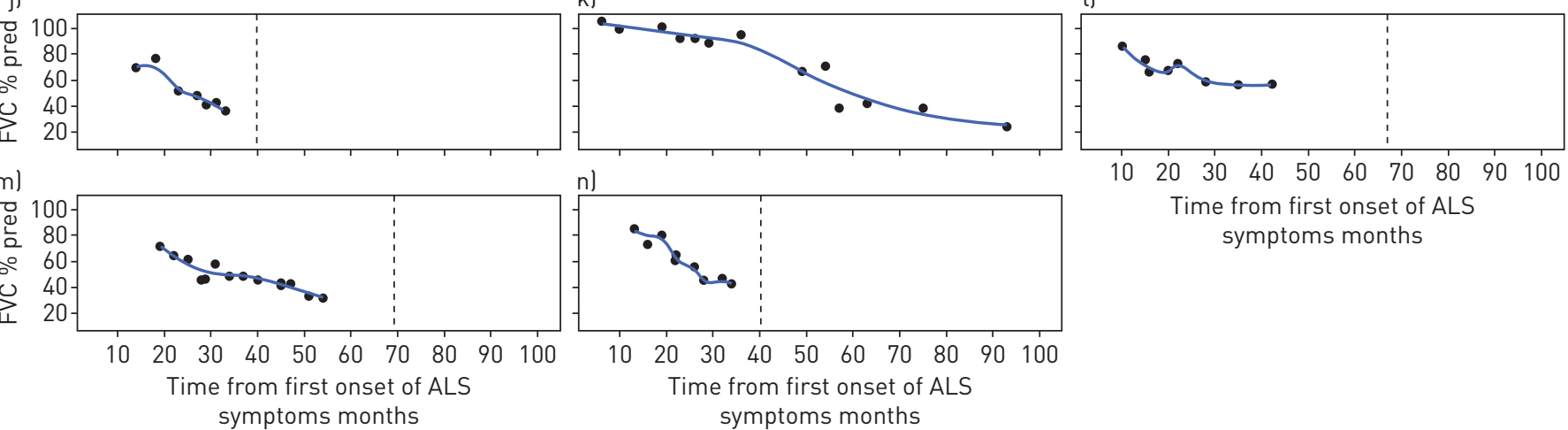

$\begin{array}{llllllllll}10 & 20 & 30 & 40 & 50 & 60 & 70 & 80 & 90 & 100\end{array}$ Time from first onset of ALS symptoms months

FIGURE 1 Plot of the pattern of decline of per cent predicted forced vital capacity in patients with amyotrophic lateral sclerosis (ALS), determined from first onset of disease (time 0 ) in selected patients (those with a larger number of spirometric observations). The figure includes a smoothing function showing that the pattern of decline in those patients demonstrates various combinations of an early plateau, a subsequent rapid decline and a late plateau of lung function, depending on when the spirometric observations were obtained in the course of the disease. The vertical bars represent death (in c) death occurred 124 months after onset; g) 125 months; k) 145 months). 


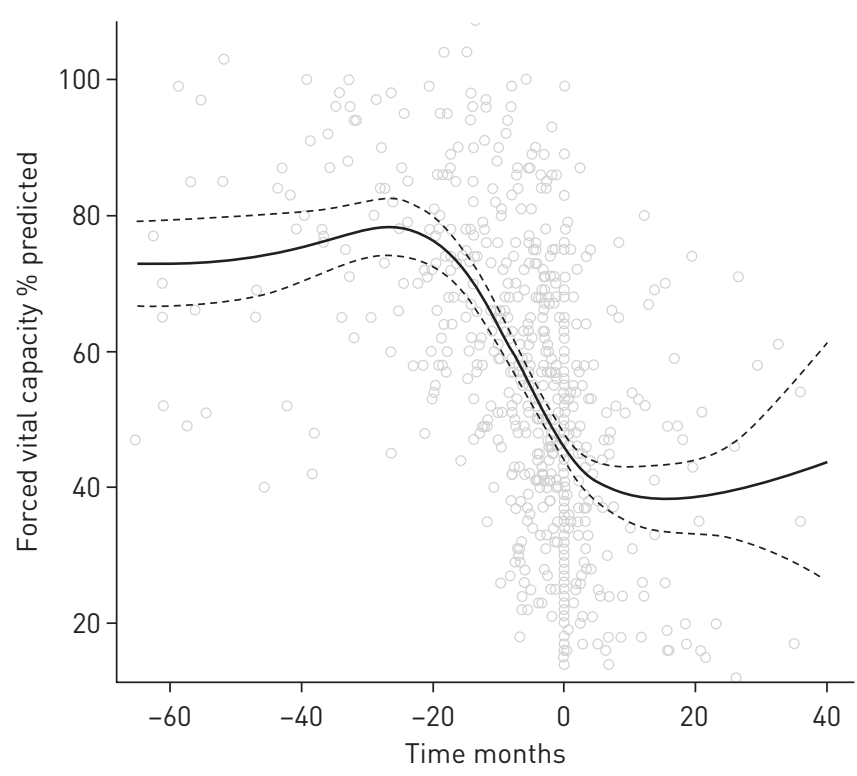

FIGURE 2 Data plot of per cent predicted forced vital capacity with nonparametric regression curve and time course centred at initiation of noninvasive positive-pressure ventilation (time 0). The fit of the course of lung function decline was done with penalised thin-plate regression. The solid curve is the estimated mean function and the dashed curves are the $95 \%$ confidence bands.

The first derivative of Equation 1 defined the slope of FVCP decline at any time $(t)$ :

$$
\frac{\mathrm{dFVCP}}{\mathrm{d} t}=\frac{b_{2} \mathrm{e}^{\frac{b_{3}+t}{b_{4}}}}{b_{4}\left(\mathrm{e}^{\frac{b_{3} b_{4}}{b^{4}}}+\mathrm{e}^{\frac{t}{b_{4}}}\right)^{2}}
$$

The same modelling was performed for MIPP. Maximisation likelihood estimation with adaptive Gaussian quadrature was applied to solve nonlinear models and estimate parameters. Statistical analyses using SAS version 9.3 software (SAS Institute, Cary, NC, USA) were performed at a significance level of 0.05 . $\mathrm{R}$ studio software was used for plots (R Project for Statistical Computing, Vienna, Austria).

\section{Results}

In total, 507 patients with probable or definite ALS were referred to the neuromuscular pulmonary clinic. Median age was 62 years (interquartile range (IQR) $53-70$ years); $44 \%$ were female, $92.5 \%$ were white, $6.3 \%$ were African-American, with additional characteristics in table E1. A total of 2392 spirometries were assessed (mean: 4.7 per patient). There were two outliers with disease onset in 1976 and 1980 that were excluded from the parametric models. Model 1 projected an initial FVCP asymptote of 100.3\% (table 1). After accelerated lung function decline, an inflection point occurred 17.2 months after disease onset at an FVCP of $63.3 \%$, after which lung function decline slowed to a plateau FVCP of $26.2 \%$.

Of the 507 patients, 4 already had a tracheostomy and invasive ventilation before the NIPPV decision, 84 were determined not to require NIPPV and continued to be followed during the course of the study, and 419 were deemed to qualify for NIPPV in the course of their follow-up visits at the pulmonary clinic. Of those, 181 were considered to be adherent on follow-up and 172 did not meet adherence criteria (see supplement for details). The remaining 66 patients had no determination of adherence made before they died, predominantly because of a distinctly short survival at a median of 3 months following the NIPPV decision (table E3). This group also had a significantly lower FVCP at initiation of NIPPV compared with the other two groups. Other characteristics are shown in the supplement (tables E2 and E3). Most notably, survival as measured from disease onset, from diagnosis or from initiation of NIPPV was significantly longer in adherent patients (table E3).

The median age of the 353 patients with known adherence was 62 years (IQR 53-70 years) and the median first FVCP after ALS diagnosis was 67\% (IQR 51-83\%). There were 1613 spirometries in this group at an average of 4.6 per patient. The median FVCP at initiation of NIPPV was 49\% (IQR 40-63\%) and the median maximum inspiratory pressure was 34.0\% (IQR 25.0-43.4\%). Model 2 (table 2 and figure 4) indicates upper and lower asymptotes for FVCP of $74.0 \%$ and $40.6 \%$, respectively. A midpoint between asymptotes occurred 5.2 months before NIPPV initiation at an FVCP of $57.3 \%$, with $46 \%$ of lung function 


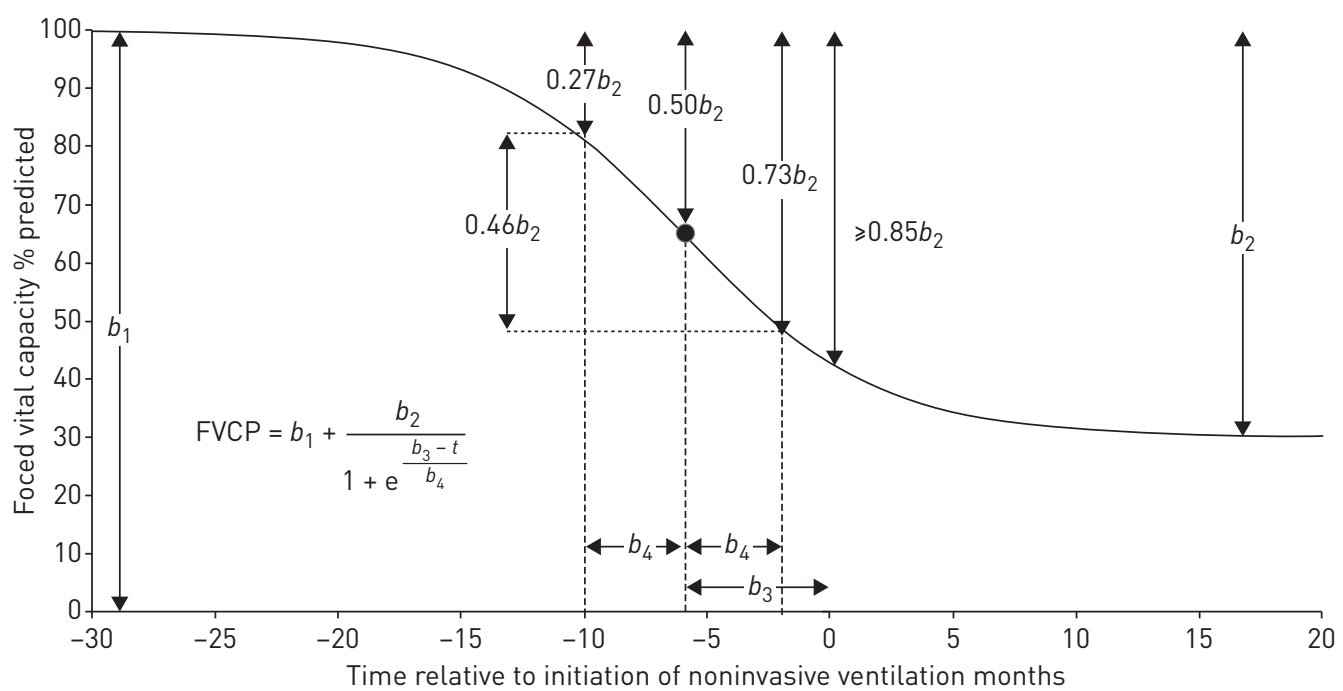

FIGURE 3 A bi-asymptotic four-parameter logistic model representing the course of per cent predicted forced vital capacity (FVCP) decline in patients with amyotrophic lateral sclerosis. The four parameters $\left(b_{1}, b_{2}, b_{3}, b_{4}\right)$ allow mathematical modelling of the bi-asymptotic pattern of decline in FVCP represented in figures 1 and 2. The time course is centred at initiation of noninvasive positive-pressure ventilation (NIPPV; time 0). The parameters, $b_{1}$ and $b_{2}$, represent lung function and $b_{3}$ and $b_{4}$ represent time; $b_{1}$ is the baseline lung function before onset of its decline (represented for demonstration purposes as an FVCP of $100 \%$ ); $b_{2}$ is the magnitude of decline to the lower asymptote (represented here as $-70 \%$ for a final FVCP of $30 \%$ ); $b_{3}$ is the time at the midpoint between the two asymptotes (represented here at 6 months prior to NIPPV), also marking the inflection point (shown as a shaded circle on the curve); and $b_{4}$ is a timescale factor (represented here as 4 months) that reflects the rapidity of decline, such that the span of decline of FVCP between time points $b_{3}+b_{4}$ and $b_{3}-b_{4}$ (8 months around the inflection point) is $46 \%$ of $b_{2}$. A smaller $b_{4}$ therefore reflects a faster lung function decline. Together, $b_{3}$ and $b_{4}$ identify key time points. In this hypothetical figure, the per cent decline relative to the total expected range of decline $\left(b_{2}\right)$ is $27 \%$ at time point $b_{3}-b_{4}$ (10 months prior to initiation of NIPPV in this representation), $50 \%$ at time point $b_{3}(6$ months prior to initiation of NIPPV in this representation), $73 \%$ at $b_{3}+b_{4}$ ( 2 months prior to initiation of NIPPV) and $\geqslant 85 \%$ at initiation of NIPPV. The corresponding values for the actual data are shown in table 3 and represented in figure 4.

decline taking place over a period of only 6.2 months around the inflection point $\left(2 b_{4}\right)$. At the time of NIPPV initiation, lung function had already declined by more than $85 \%$ of the total range between asymptotes (table 3 and figures 2 and 4). The slope of FVCP decline was $2.69 \%$ per month at the inflection, decreasing to $1.43 \%$ per month by NIPPV initiation (table 3 ).

The median age of adherent and nonadherent patients was 60.0 (IQR 53-68) and 64.0 (IQR 53-70.8) years, respectively $(\mathrm{p}=0.15)$. Those with an initial bulbar score on the revised ALS Functional Rating Scale of $>7$ (best score: 12) were more likely to be adherent than those with a score $\leqslant 7$ ( $60 \%$ versus $44 \%$, respectively; $\mathrm{p}=0.03$ ). There was no difference in FVCP between adherent and nonadherent patients, either at the upper asymptote $(72.2 \%$ and $76.1 \%$, respectively; $\mathrm{p}=0.08)$ or at the lower asymptote $(41.9 \%$

\section{TABLE 1 Parameter estimates for the course of decline of per cent predicted forced vital} capacity from onset of disease (Model 1)

\begin{tabular}{|c|c|c|c|}
\hline \multirow[t]{2}{*}{ Parameter } & \multirow[t]{2}{*}{ Significance of parameter } & \multicolumn{2}{|c|}{ Overall cohort } \\
\hline & & Mean (SE) ${ }^{\#}$ & $95 \% \mathrm{Cl}$ \\
\hline$b_{1} \%$ & Initial vital capacity & $100.3(3.8)$ & $92.8,107.9$ \\
\hline$b_{2} \%$ & Decline from initial vital capacity & $-74.1(4.6)$ & $-83.2,-65.0$ \\
\hline$b_{3}$ months & $\begin{array}{l}\text { Midpoint between initial and final vital capacity } \\
\text { (from disease onset) }\end{array}$ & $17.2(1.5)$ & $14.3,20.2$ \\
\hline$b_{4}$ months & Timescale factor representing rapidity of decline & $13.3(1.1)$ & $11.2,15.4$ \\
\hline$b_{1}+b_{2} \%$ & Final vital capacity & $26.2(2.1)$ & $22.1,30.4$ \\
\hline
\end{tabular}


TABLE 2 Parameter estimates for the course of decline of per cent predicted forced vital capacity from time of initiation of noninvasive ventilation (Model 2)

\begin{tabular}{|c|c|c|c|c|c|c|c|}
\hline \multirow[t]{2}{*}{ Parameter } & \multicolumn{2}{|c|}{ Overall cohort } & \multicolumn{2}{|c|}{ Adherent patients } & \multicolumn{2}{|c|}{ Nonadherent patients } & \multirow[t]{2}{*}{ p-value $\#$} \\
\hline & Mean (SE) ๆ & $95 \% \mathrm{Cl}$ & Mean (sE) ${ }^{\Uparrow}$ & $95 \% \mathrm{Cl}$ & Mean (SE) $)^{\Uparrow}$ & $95 \% \mathrm{Cl}$ & \\
\hline$b_{1} \%$ & $74.0(1.2)$ & $71.1,76.4$ & $72.2(1.5)$ & $69.3,75.1$ & $76.1(1.6)$ & $72.8,79.3$ & 0.081 \\
\hline$b_{3}$ months & $-5.2(0.5)$ & $-6.2,-4.3$ & $-4.3(0.5)$ & $-5.3,3.3$ & $-6.7(0.9)$ & $-8.4,-5.0$ & 0.017 \\
\hline$b_{4}$ months & $3.1(0.4)$ & $2.4,3.9$ & $2.3(0.4)$ & $1.4,3.1$ & $3.8(0.6)$ & $2.6,5.0$ & 0.036 \\
\hline$b_{1}+b_{2} \%$ & $40.6(2.1)$ & $34.7,43.0$ & $41.9(1.1)$ & $39.7,44.1$ & $38.5(2.1)$ & $34.4,42.7$ & 0.16 \\
\hline
\end{tabular}

and $38.5 \%$, respectively; $\mathrm{p}=0.16$ ). However, the inflection point (midway between the asymptotes) occurred 4.3 months before NIPPV initiation in subsequently adherent patients, compared with 6.7 months before NIPPV initiation in subsequently nonadherent patients $(\mathrm{p}=0.017)$. Additionally, $46 \%$ of lung function decline between asymptotes occurred over $4.6(2 \times 2.3)$ months in subsequently adherent patients, but over $7.6(2 \times 3.8)$ months in subsequently nonadherent patients ( $p=0.036$ for $b 4$ parameter comparison). In essence, the monthly rate of loss of FVCP at the inflection point was faster in subsequently adherent patients than nonadherent patients (3.35\% versus $2.35 \%$, respectively) (table 3 ). The course of forced expiratory volume in $1 \mathrm{~s}$ decline followed the same pattern with similar parameters (Table E4).

The FVCP at the time of NIPPV initiation was $45.9 \%$ in the model in both adherent and nonadherent groups. At that time, the monthly slope of FVCP decline was $1.50 \%$ in adherent patients versus $1.17 \%$ in nonadherent patients (table 3).

The course of decline of MIPP with a smaller number of observations (2.95 per patient) was also bi-asymptotic (table 4 and figure E2). In linear mixed-model analyses, there was a linear relationship between SNIP and FVCP, and between the ALSFRS-R and FVCP (see supplement).

\section{Discussion}

This study tracked FVCP from the initial ALS diagnosis and showed that after the initial stable levels, FVCP decline accelerated before reaching an inflection point, and then significantly slowed. A bi-asymptotic pattern with a final plateau was suggested in individual tracings, a nonparametric representation of the spirometry data and in parametric models. Overall, $46 \%$ of the total range of decline in FVCP took place

TABLE 3 Per cent total decline in forced vital capacity per cent predicted (FVCP) relative to the total range, and slope of decline at various key time points

\begin{tabular}{|c|c|c|c|c|c|}
\hline $\begin{array}{l}\text { Time relative to NIPPV } \\
\text { start (months) }\end{array}$ & $\begin{array}{l}\text { Per cent total } \\
\text { decline }\end{array}$ & $\begin{array}{l}\text { FVCP in } \\
\text { overall cohort }\end{array}$ & $\begin{array}{l}\text { Change in } \mathrm{FVCP} / \text { month } \\
\text { in overall cohort }\end{array}$ & $\begin{array}{c}\text { Change in FVCP/month } \\
\text { (adherent) }\end{array}$ & $\begin{array}{c}\text { Change in FVCP/month } \\
\text { (nonadherent) }\end{array}$ \\
\hline$\sim 30$ & 0 & 74.0 & 0 & 0 & 0 \\
\hline$b_{3}$ & 50 & 57.3 & -2.69 & -3.35 & -2.35 \\
\hline$b_{3}+b_{4}$ & 73 & 49.6 & -2.11 & -2.64 & -1.85 \\
\hline 0 & $\geqslant 85$ & 45.9 & -1.43 & -1.50 & -1.17 \\
\hline \multicolumn{6}{|c|}{$\begin{array}{l}b_{3} \text { : time (months) to the midpoint between the two asymptotes, also representing the inflection point of the FVCP time-course curve; } b_{4} \text { : } \\
\text { timescale factor (months) that reflects the rapidity of decline of forced vital capacity, such that a smaller } b_{4} \text { reflects a more rapid decline } \\
\text { Slopes are derived from the first derivative of vital capacity with time of the following equation: FVCP }=b_{1}+b_{2} /\left(1+\mathrm{e}^{\left(\left(b_{3}-\mathrm{t}\right) / b_{4}\right)} \text { such tha }\right. \\
\text { dFVCP }\end{array}$} \\
\hline \multicolumn{6}{|c|}{$\frac{\mathrm{dt}}{b_{4}\left(\mathrm{e}^{\left(b_{3} / b_{4}\right)}+\mathrm{e}^{\left.\left(t / b_{4}\right)\right)^{2}}\right.}$} \\
\hline
\end{tabular}




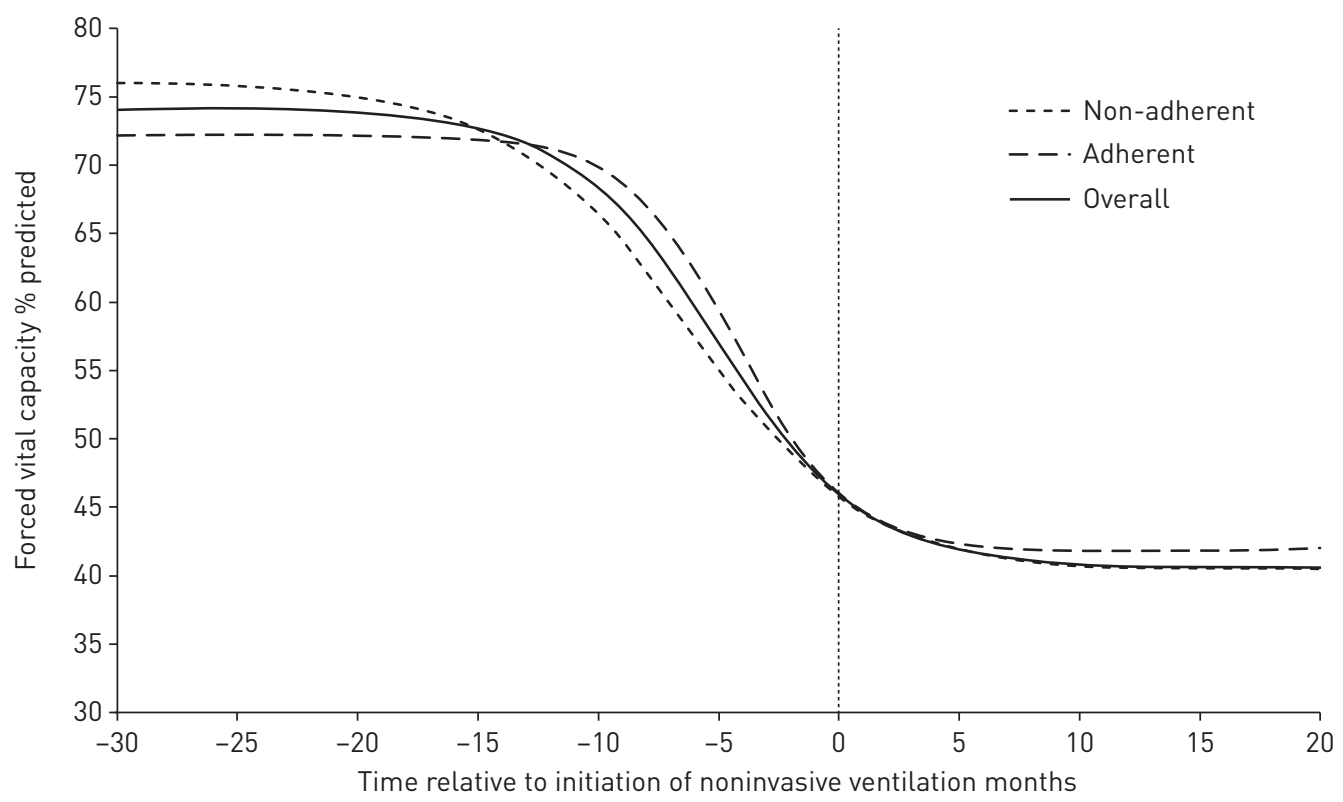

FIGURE 4 Plot of the course of per cent predicted forced vital capacity decline in amyotrophic lateral sclerosis after resolution of individual parameters using a nonlinear mixed-model regression analysis. Includes overall patients and patients stratified by adherence to noninvasive positive-pressure ventilation. The time course is centred at initiation of noninvasive positive-pressure ventilation (time 0).

over 6.2 months, and NIPPV was initiated when at least $85 \%$ of the total decline had already occurred, with no difference in initial or final FVCP between patients adherent or nonadherent to NIPPV. Differences in time factors reflecting the pattern of decline were evident before NIPPV initiation: nonadherent patients lost $50 \%$ of lung function ahead of adherent patients. Furthermore, the rate of decline was significantly faster, and fewer bulbar symptoms were noted in patients subsequently classified as adherent.

This study reconciles descriptions of the course of lung function decline as accelerated [11], stable [12-14] or decelerating [15-17], depending on the timing and duration of the observations. The speed of lung function decline (small $b_{4}$ parameter) may inform follow-up schedules and justify re-evaluations at 4-6-month intervals. Finally, current NIPPV guidelines [8], though useful in improving survival and quality of life $[7,15]$, initiate NIPPV when at least $85 \%$ of the total decline has already occurred. While guidelines in the Unites States and Europe differ, the median time from disease onset to NIPPV is comparable at 21 months (IQR 13-33 months) in our study, compared with 24 months (IQR 19-57 months) using European guidelines [10].

Although some attribute a slowing in FVCP decline to NIPPV initiation [5, 18-21], this study concords with others that show that deceleration is independent of NIPPV [15-17]. Therefore, comparing the rate of decline pre- and post-NIPPV when started after the inflection may incorrectly convey a beneficial effect

\section{TABLE 4 Parameter estimates for the course of decline of per cent predicted maximum}

inspiratory pressure

\begin{tabular}{llll} 
Parameter & Mean (SE) & $\mathbf{9 5 \% ~ C l}$ & p-value \\
\hline $\boldsymbol{b}_{\mathbf{1}} \%$ & $58.1(3.7)$ & $50.9,65.3$ & $<0.001$ \\
$\boldsymbol{b}_{\mathbf{2}} \%$ & $-27.8(4.5)$ & $-36.6,-19.1$ & $<0.001$ \\
$\boldsymbol{b}_{\mathbf{3}}$ months & $-7.8(1.6)$ & $-10.8,-4.7$ & $<0.001$ \\
$\boldsymbol{b}_{\mathbf{4}}$ months & $3.7(1.2)$ & $1.3,6.1$ & 0.0027
\end{tabular}

$b_{1}$ : per cent predicted maximum inspiratory pressure before onset of decline of lung function; $b_{2}$ : magnitude of decline in predicted maximum inspiratory pressure to the lower asymptote, represented in the model as a negative number; $b_{3}$ : time (months) relative to initiation of noninvasive ventilation to the midpoint between the two asymptotes, also representing the inflection point of the per cent predicted maximum inspiratory pressure; $b_{4}$ : a timescale factor (months) that reflects the rapidity of decline of per cent predicted maximum inspiratory pressure. 
to the intervention. Similarly, as an accelerated rate of decline of lung function partly predicted future NIPPV adherence, observational studies may erroneously conclude that NIPPV slows the rate of decline.

Our study was limited by the subjective definition of adherence, the long duration of the study with potential for variations in practice, projections based on mathematical modelling, survival bias and the use of FVCP as a marker of disease progression.

Most patients in this study started NIPPV before the widespread availability of device downloads, and objective adherence criteria (i.e. at least $4 \mathrm{~h}$ of use on at least $70 \%$ of days) differ from our original definition (i.e. at least $4 \mathrm{~h}$ consecutively of use during sleep). Most of our results and conclusions are unaffected by objective characterisation of adherence, and findings that depend on adherence determination concord with the literature. For instance, our finding that an accelerated rate of decline predicts NIPPV adherence, suggests that respiratory muscle involvement is a predominant source of symptoms, and is consistent with studies that identified orthopnoea and dyspnoea as predictors of NIPPV adherence [18, 30]. We also noted an association between preserved bulbar function and adherence [6, 20, 31].

Changes occurred in our practice after 2014 to refine the NIPPV settings with an in-office review of detailed device downloads and end-tidal $\mathrm{CO}_{2}$. Until then, our practice was uniform for the duration of this current study, with the same two physicians, using the same criteria for initiation of NIPPV and with a standardised approach of care including secretion management.

Model 2 concords with several key observations. For example, the model's estimate of FVCP at NIPPV initiation matches the actual number ( $45.9 \%$ versus $49.0 \%$, respectively; tables 3 and E3, and figure 4 ). The monthly drop in FVCP at the inflection point is $2.69 \%$ in the model (table 3 ), which concords rather precisely with results from large ALS clinical trials $[12,32]$. Our model agrees with figure 1 (which is not subject to a survival bias) and with the nonparametric representation in figure 2 (which is not biased by the assumptions of the mathematical model). The lower line of the 95th percentile confidence band in figure 2 shows at least a significant slowing of the lung function decline. There was no particular bias of loss of potential forced vital capacity measurements in patients with significant late bulbar symptoms compared with those without, and the median time from the last FVCP measurement to death was 4.7 months (fig. 1 and supplement). While this analysis does not address the quality of the measurements in bulbar patients, we noted a more accelerated decline of lung function in adherent patients who had an overall better-preserved bulbar function. Overall, we believe that the lower asymptote is less likely to represent a bias of survival of patients who can still perform the spirometry manoeuvre.

Vital capacity and its rate of decline are associated with clinically meaningful outcomes and remain essential measures of disease progression $[2,12]$. A caveat is that the bi-asymptotic pattern may reflect the sigmoid relationship of lung volume versus pressure [33]. For instance, the FVCP can remain normal despite significant impairment in respiratory muscle strength and may be limited in its ability to detect respiratory insufficiency [5]. However, the MIPP also followed a bi-asymptotic pattern of decline with the same time course as FVCP (table 4). Specifically, the 95\% confidence intervals for time parameters b3 and b4 of FVCP decline in table 2 are encompassed in the same parameters for the MIPP in table 4 . We also noted a linear relationship between SNIP or ALSFRS-R and FVCP in a more limited set of data.

These results suggest a parallel decline of strength and volume, and that factors unrelated to an artefact of the pressure-volume relationship mitigate lung function decline. These potential factors include the presence of disease-resistant motor neurons [34] and the sprouting of adjacent nerve terminals to re-innervate degenerating neuromuscular junctions with the formation of enlarged motor units [35-37]. These re-innervations fire inconsistently with frequently blocked conduction [36], and may be vulnerable to neuromuscular activity (and potential respiratory muscle overload) which reduce re-innervations [38] and promote loss of the enlarged motor units [39]. This may explain the survival disadvantage imparted to diaphragm pacing in ALS [40,41]. In contrast, NIPPV offloads the work of breathing and reduces diaphragmatic electromyography activity [42], though with a concern that complete diaphragm inactivity may cause muscle atrophy [43]. Whether earlier initiation of NIPPV would maintain re-innervations and reduce dropout of enlarged motor units is unclear. However, if such a benefit is to be realised, it would require earlier initiation of NIPPV, perhaps prior to the inflection point (which may signal the increasing influence of mitigating factors), which corresponds in our models to an FVCP $\geqslant 60 \%$ (table 3) approximately 17 months after disease onset (table 1).

Several studies have documented the feasibility of early NIPPV initiation, with benefits in survival [44], quality of life [45] and lung function decline [46, 47]. In a randomised trial, NIPPV at a mean FVCP of $74 \%$ slowed FVCP decline by $0.44 \%$ per month compared with sham therapy [47]. From a practical perspective, clinicians deliberating early NIPPV should balance risks and benefits. Adherence may be poor at $<4 \mathrm{~h}$ per night with early implementation of NIPPV [47], or in the presence of significant bulbar 
symptoms [6, 20, 31]. Furthermore, mucus accumulation portends a poor prognosis with NIPPV [48], and NIPPV is potentially associated with deaths from bronchopneumonia [4]. However, the presence of bulbar symptoms does not preclude the use of NIPPV. For instance, NIPPV in the context of significant bulbar symptoms may still impart a survival advantage [49] and a quality-of-life advantage [7]. One study even reported a greater survival benefit of NIPPV in bulbar-onset ALS relative to patients with nonbulbar onset [21]. The latter findings may reflect that NIPPV is initiated earlier in bulbar-onset ALS [21, 32].

Our study shows a pattern of rapid decline of lung function in patients with ALS, followed by a slowing of decline to a potential plateau. Differences between adherent and nonadherent patients occur before NIPPV initiation, suggesting that differentiators such as onset of decline and rapidity of decline predict adherence to NIPPV rather than being effects of NIPPV. Current reimbursement criteria initiate NIPPV well after lung function has significantly declined and may be too late to affect the course of the disease. European guidelines, which combine clinical signs with expanded respiratory function tests (including FVCP $<80 \%$ ) to initiate NIPPV, may be more appropriate $[1,9,10]$.

Acknowledgments: Thanks to the patients and their caregivers for providing the data used in this study, and to the nurses and respiratory technicians who performed the pulmonary function testing. These data were previously presented at the American Thoracic Society meetings in Denver, CO, USA (2015) and San Francisco, CA, USA (2016).

Author contributions: T.S. Panchabhai, E. Mireles Cabodevila and E.P. Pioro contributed substantially to the study design, data collection, interpretation of analysis and writing of the manuscript. X. Wang and X. Han contributed substantially to the statistical analysis, interpretation of the analysis and writing the corresponding sections of the manuscript. L.S. Aboussouan contributed substantially to the study design, data collection, analysis of results, interpretation of analysis and the manuscript writing, had full access to all of the data in the study, and takes responsibility for the integrity of the data.

Conflict of interest: T.S. Panchabhai has nothing to disclose. E. Mireles Cabodevila reports book royalties from Bartlett and Jones Publishers, honoraria for a book chapter and questions from the American College of Physicians and honoraria for lectures from Dong'e People's Hospital, China, outside the submitted work. E.P. Pioro reports grants from the NIH/CDC, the ALS Association and Iron Horse Diagnostics, Inc., and personal fees from Avanir Pharmaceuticals, Inc., Cytokinetics, Inc., ITF Pharma, Inc., and MT Pharma America, Inc., outside the submitted work. X. Wang has nothing to disclose. X. Han has nothing to disclose. L.S. Aboussouan reports personal fees from UpToDate Wolters Kluwer outside the submitted work.

\section{References}

1 Andersen PM, Abrahams S, Borasio GD, et al. EFNS guidelines on the clinical management of amyotrophic lateral sclerosis (MALS) - revised report of an EFNS task force. Eur J Neurol 2012; 19: 360-375.

2 Westeneng HJ, Debray TPA, Visser AE, et al. Prognosis for patients with amyotrophic lateral sclerosis: development and validation of a personalised prediction model. Lancet Neurol 2018; 17: 423-433.

3 Brown RH, Al-Chalabi A. Amyotrophic lateral sclerosis. N Engl J Med 2017; 377: 162-172.

4 Burkhardt C, Neuwirth C, Sommacal A, et al. Is survival improved by the use of NIV and PEG in amyotrophic lateral sclerosis (ALS)? A post mortem study of 80 ALS patients. PLoS ONE 2017; 12: e0177555.

5 Miller RG, Jackson CE, Kasarskis EJ, et al. Practice parameter update: the care of the patient with amyotrophic lateral sclerosis: drug, nutritional, and respiratory therapies (an evidence-based review): report of the Quality Standards Subcommittee of the American Academy of Neurology. Neurology 2009; 73: 1218-1226.

6 Aboussouan LS, Khan SU, Meeker DP, et al. Effect of noninvasive positive-pressure ventilation on survival in amyotrophic lateral sclerosis. Ann Intern Med 1997; 127: 450-453.

7 Bourke SC, Tomlinson M, Williams TL, et al. Effects of non-invasive ventilation on survival and quality of life in patients with amyotrophic lateral sclerosis: a randomised controlled trial. Lancet Neurol 2006; 5: 140-147.

8 Sunwoo BY, Mulholland M, Rosen IM, et al. The changing landscape of adult home noninvasive ventilation technology, use, and reimbursement in the United States. Chest 2014; 145: 1134-1140.

9 National Institute for Health and Care Excellence. Motor neurone disease: assessment and management. www.nice.org.uk/guidance/ng42 Date last accessed: March 23, 2019. Date last updated: 2016.

10 Georges M, Golmard JL, Llontop C, et al. Initiation of non-invasive ventilation in amyotrophic lateral sclerosis and clinical practice guidelines: single-centre, retrospective, descriptive study in a national reference centre. Amyotroph Lateral Scler Frontotemporal Degener 2017; 18: 46-52.

11 Fallat RJ, Jewitt B, Bass M, et al. Spirometry in amyotrophic lateral sclerosis. Arch Neurol 1979; 36: 74-80.

12 Andrews JA, Meng L, Kulke SF, et al. Association between decline in slow vital capacity and respiratory insufficiency, use of assisted ventilation, tracheostomy, or death in patients with amyotrophic lateral sclerosis. JAMA Neurol 2018; 75: 58-64.

13 Armon C, Graves MC, Moses D, et al. Linear estimates of disease progression predict survival in patients with amyotrophic lateral sclerosis. Muscle Nerve 2000; 23: 874-882.

14 Baumann F, Henderson RD, Morrison SC, et al. Use of respiratory function tests to predict survival in amyotrophic lateral sclerosis. Amyotroph Lateral Scler 2010; 11: 194-202.

15 Aboussouan LS, Khan SU, Banerjee M, et al. Objective measures of the efficacy of noninvasive positive-pressure ventilation in amyotrophic lateral sclerosis. Muscle Nerve 2001; 24: 403-409.

16 Clavelou P, Blanquet M, Peyrol F, et al. Rates of progression of weight and forced vital capacity as relevant measurement to adapt amyotrophic lateral sclerosis management for patient. Result of a French multicentre cohort survey. J Neurol Sci 2013; 331: 126-131.

17 Schiffman PL, Belsh JM. Pulmonary function at diagnosis of amyotrophic lateral sclerosis. Rate of deterioration. Chest 1993; 103: 508-513. 
18 Bourke SC, Bullock RE, Williams TL, et al. Noninvasive ventilation in ALS: indications and effect on quality of life. Neurology 2003; 61: 171-177.

19 Kleopa KA, Sherman M, Neal B, et al. Bipap improves survival and rate of pulmonary function decline in patients with ALS. J Neurol Sci 1999; 164: 82-88.

20 Lo Coco D, Marchese S, Pesco MC, et al. Noninvasive positive-pressure ventilation in ALS: predictors of tolerance and survival. Neurology 2006; 67: 761-765.

21 Berlowitz DJ, Howard ME, Fiore JF, Jr., et al. Identifying who will benefit from non-invasive ventilation in amyotrophic lateral sclerosis/motor neurone disease in a clinical cohort. J Neurol Neurosurg Psychiatr 2016; 87: 280-286.

22 Brooks BR. El Escorial World Federation of Neurology criteria for the diagnosis of amyotrophic lateral sclerosis. J Neurol Sci 1994; 124: Suppl., 96-107.

23 Cedarbaum JM, Stambler N, Malta E, et al. The ALSFRS-R: a revised ALS functional rating scale that incorporates assessments of respiratory function. BDNF ALS Study Group (phase III). J Neurol Sci 1999; 169: 13-21.

24 Hankinson JL, Odencrantz JR, Fedan KB. Spirometric reference values from a sample of the general US population. Am J Respir Crit Care Med 1999; 159: 179-187.

25 Miller MR, Hankinson J, Brusasco V, et al. Standardisation of spirometry. Eur Respir J 2005; 26: 319-338.

26 American Thoracic Society/European Respiratory Society. ATS/ERS Statement on respiratory muscle testing. Am J Respir Crit Care Med 2002; 166: 518-624.

27 Black LF, Hyatt RE. Maximal respiratory pressures: normal values and relationship to age and sex. Am Rev Respir Dis 1969; 99: 696-702

28 Wood SN. Generalized additive models: an introduction with R. Boca Raton, Chapman and Hall/CRC, 2006.

29 Pinheiro JC, Bates DM. Mixed-effects models in S and S-PLUS. New York, Springer, 2000.

30 Jackson CE, Lovitt S, Gowda N, et al. Factors correlated with NPPV use in ALS. Amyotroph Lateral Scler 2006; 7: $80-85$.

31 Gruis KL, Brown DL, Schoennemann A, et al. Predictors of noninvasive ventilation tolerance in patients with amyotrophic lateral sclerosis. Muscle Nerve 2005; 32: 808-811.

32 Thakore NJ, Lapin BR, Pioro EP, et al. Variation in noninvasive ventilation use in amyotrophic lateral sclerosis. Neurology 2019; 93: e306-e316.

33 Jonson B, Svantesson C. Elastic pressure-volume curves: what information do they convey? Thorax 1999; 54 $82-87$.

34 Nijssen J, Comley LH, Hedlund E. Motor neuron vulnerability and resistance in amyotrophic lateral sclerosis. Acto Neuropathol 2017; 133: 863-885.

35 Martineau E, Di Polo A, Vande Velde C, et al. Dynamic neuromuscular remodeling precedes motor-unit loss in a mouse model of ALS. eLife 2018; 7: e41973.

36 Stålberg EV, Sonoo M. Assessment of variability in the shape of the motor unit action potential, the "jiggle," at consecutive discharges. Muscle Nerve 1994; 17: 1135-1144.

37 Yuen EC, Olney RK. Longitudinal study of fiber density and motor unit number estimate in patients with amyotrophic lateral sclerosis. Neurology 1997; 49: 573-578.

38 Tam SL, Archibald V, Jassar B, et al. Increased neuromuscular activity reduces sprouting in partially denervated muscles. J Neurosci 2001; 21: 654-667.

39 Tam SL, Archibald V, Tyreman N, et al. Effect of exercise on stability of chronically enlarged motor units. Muscle Nerve 2002; 25: 359-369.

40 Gonzalez-Bermejo J, Morelot-Panzini C, Tanguy ML, et al. Early diaphragm pacing in patients with amyotrophic lateral sclerosis (RespiStimALS): a randomised controlled triple-blind trial. Lancet Neurol 2016; 15: 1217-1227.

41 DiPALS Writing Committee, DiPALS Study Group Collaborators. Safety and efficacy of diaphragm pacing in patients with respiratory insufficiency due to amyotrophic lateral sclerosis (DiPALS): a multicentre, open-label, randomised controlled trial. Lancet Neurol 2015; 14: 883-892.

42 Kallet RH, Diaz JV. The physiologic effects of noninvasive ventilation. Respir Care 2009; 54: 102-115.

43 Levine S, Nguyen T, Taylor N, et al. Rapid disuse atrophy of diaphragm fibers in mechanically ventilated humans. N Engl J Med 2008; 358: 1327-1335.

44 Lechtzin N, Scott Y, Busse AM, et al. Early use of non-invasive ventilation prolongs survival in subjects with ALS Amyotroph Lateral Scler 2007; 8: 185-188.

45 Jackson CE, Rosenfeld J, Moore $\mathrm{DH}$, et al. A preliminary evaluation of a prospective study of pulmonary function studies and symptoms of hypoventilation in ALS/MND patients. J Neurol Sci 2001; 191: 75-78.

46 Elamin EM, Wilson CS, Sriaroon C, et al. Effects of early introduction of non-invasive positive pressure ventilation based on forced vital capacity rate of change: variation across amyotrophic lateral sclerosis clinical phenotypes. Int J Clin Pract 2019; 73: e13257.

47 Jacobs TL, Brown DL, Baek J, et al. Trial of early noninvasive ventilation for ALS: a pilot placebo-controlled study. Neurology 2016; 87: 1878-1883.

48 Peysson S, Vandenberghe N, Philit F, et al. Factors predicting survival following noninvasive ventilation in amyotrophic lateral sclerosis. Eur Neurol 2008; 59: 164-171.

49 Sancho J, Martinez D, Bures E, et al. Bulbar impairment score and survival of stable amyotrophic lateral sclerosis patients after noninvasive ventilation initiation. ERJ Open Res 2018; 4: 00159-02017. 